\title{
RELACIÓN ENTRE ESTRÉS POSTRAUMÁTICO, AFRONTAMIENTO REPRESIVO Y RUMIACIÓN EN ESTUDIANTES UNIVERSITARIOS CHILENOS
}

\author{
THE RELATIONSHIP BETWEEN POST-TRAUMATIC \\ STRESS, REPRESSIVE COPING AND RUMINATION AMONG \\ CHILEAN UNIVERSITY STUDENTS
}

\author{
Pamela Paz Caro Monroy * \\ FÉlix Mario Cova Solar ${ }^{* *}$
}

\begin{abstract}
RESUMEN
Los desastres naturales pueden tener distinto tipo de impactos en el bienestar psicológico y salud mental. Una de las respuestas que se ha asociado con mayor frecuencia a los desastres es la denominada sintomatología postraumática. El impacto psicológico de los desastres naturales está influido por distintos factores. Un factor individual que probablemente puede influir es la forma de afrontamiento empleada por cada persona. Objetivo: La presente investigación evaluó la relación entre estrés postraumático y dos formas de afrontamiento, el estilo represivo y la rumiación. Material y método: muestra de 314 estudiantes universitarios que vivieron el terremoto y tsunami del 27 de febrero de 2010 en la zona centro-sur de Chile, quienes respondieron una batería de instrumentos de autoinforme. Se realizó análisis bivariado y multivariado. Resultados: Se observaron asociaciones inversas entre el afrontamiento represivo y estrés postraumático y asociaciones directas entre rumiación y estrés postraumático. El afrontamiento represivo y la rumiación mostraron ser variables con una relación inversa entre sí. Conclusiones: Estos resultados son de interés para una mejor comprensión de la relación entre el tipo de respuesta de afrontamiento empleadas por las personas y su susceptibilidad a responder en forma negativa a situaciones traumáticas como los desastres naturales. El afrontamiento represivo puede no ser una respuesta negativa y podría contribuir a una menor rumiación, la que sí muestra ser un factor que se asocia a una mayor sintomatología postraumática.
\end{abstract}

Palabras clave: Trastorno por estrés postraumático, afrontamiento represivo, rumiación, desastres naturales.

\begin{abstract}
Natural disasters have different impacts on people's psychological well-being and mental health. One of the most frequent consequences is the post-traumatic symptomatology. The psychological impact of disasters might be influenced by several factors. The coping style is one of the personal factors that influence the psychological impact of disasters. Objective: The current research assessed the relationship between posttraumatic stress and two ways of coping, repressive style and rumination in a sample of 314 undergraduate students that lived the earthquake and tsunami in the central region of Chile and that answered self-informed tools. Results: An inverse relationship between repressive coping and post-traumatic stress was found, as well as direct relationship between rumination and post-traumatic stress. Repressive coping and rumination showed an inverse relationship. Conclusion: These results allow a better understanding of the relationship between
\end{abstract}

\footnotetext{
*Enfermera, Docente Universidad San Sebastián, Concepción, Chile, Email: pamela.caro@uss.cl

** Psicólogo, Docente Depto. Psicología Universidad de Concepción, Concepción, Chile, Email: ecova@udec.cl
} 
coping responses and person's propensity to answer negatively to traumatic experiences such as natural disasters. Repressive coping may not be a negative response and so could contribute to a decreased rumination, which is a factor that is associated to a greater post-traumatic symptomatology.

Key words: Stress disorders, Post-traumatic, repressive coping, rumination, natural disasters.

Fecha recepción: 19/08/11 Fecha aceptación: 03/12/12

\section{INTRODUCCIÓN}

Los desastres naturales pueden afectar significativamente la vida de comunidades y personas. En el año 2003, una de cada 25 personas fue afectada por algún desastre. Se estima que los desastres naturales podrían aumentar su frecuencia e impacto por factores como el cambio climático, el crecimiento poblacional, la urbanización y la pobreza (1, 2).

En general, las respuestas psicológicas frente a los desastres han sido conceptualizadas como "respuestas traumáticas". El concepto de trauma alude a eventos peligrosos y repentinos que abruman los recursos psicológicos, físicos y económicos de las personas y comunidades (3). Aun cuando las consecuencias psicológicas de los desastres son diversas, el trastorno de estrés postraumático (TEPT) es la patología más comúnmente estudiada después de un desastre (4). Revisiones recientes de trastornos psicopatológicos ligados a desastres señalan que el TEPT sería la consecuencia más observada y probablemente la más discapacitante de los desastres, aun cuando existe considerable debate al respecto (5).

El trastorno de estrés postraumático (TEPT) es un patrón de síntomas característicos que pueden seguir a la exposición a eventos que representan un peligro real para la vida o integridad física de la persona. Estos síntomas incluyen distintas formas de re-experimentación de la experiencia, conductas de evitación e hiperactivación fisiológica (6).

Reconocer factores relacionados con la respuesta emocional a las situaciones traumáticas, y a los desastres en particular es de importancia para establecer lineamientos y estrategias de promoción, prevención y tratamiento.

El estilo de afrontamiento represivo fue definido por Weinberger et al. (7) como una forma de respuesta caracterizada por el no reconocimiento por parte de las personas de sus respuestas afectivas. Se ha operacionalizado a través de la combinación de puntuaciones bajas en una medida del rasgo ansiedad y altas puntuaciones en deseabilidad social $(7,8)$. Pese a que habitualmente el afrontamiento represivo ha sido considerado un estilo desadaptativo, investigaciones recientes lo han identificado como un factor que puede tener un efecto protector a corto y largo plazo en situaciones de estrés (9-11).

La rumiación se conceptualiza como el pensamiento pasivo, repetitivo sobre el malestar anímico y depresión que se estén experimentando, y sobre sus posibles causas y consecuencias (12). Se ha observado consistentemente su relación con la presencia y desarrollo de sintomatología depresiva y, también, ansiosa (13). Algunos estudios también han mostrado que se relaciona con la presencia y desarrollo de estrés postraumático (1416). En los últimos años se han identificado dos dimensiones distintas en la rumiación: reflexión y rumiación negativa. La reflexión tiene que ver con el análisis de las conductas vividas. La rumiación negativa se asocia con una forma específica de la rumiación, que se centra en un pensamiento con valencia emocional negativa focalizado en el malestar experimentado y que se asocia a una evalua- 
ción negativa de sí mismo (13).

El estilo afrontamiento represivo y rumiador pueden ser vistos como opuestos en alguna medida; sin embargo, ello ha sido muy poco explorado empíricamente (17).

El desarrollo de sintomatología postraumática frente a eventos traumáticos está influido por muy diversos factores. Uno de ellos es el género. Consistentemente las investigaciones muestran mayor prevalencia de sintomatología postraumática en mujeres $(4,18)$. También se observan diferencias de género respecto del empleo de afrontamiento represivo, más frecuente en hombres ( 9 , 11) y de la rumiación, más frecuente en mujeres (12-15).

La zona centro sur de Chile vivió un terremoto de gran magnitud el 27 de febrero de 2010: Zonas costeras fueron afectadas además por un tsunami. A propósito de ese evento, esta investigación buscó identificar factores relacionados con el desarrollo de sintomatología postraumática en estudiantes universitarios que se vieron expuestos a este desastre, e identificar su relación con dos tipos de estrategias de afrontamiento: el estilo de afrontamiento represivo y la rumiación.

En atención a estos antecedentes este estudio se propuso evaluar las siguientes hipótesis: 1) el afrontamiento represivo se relaciona con menor presencia de sintomatología postraumática; 2) la rumiación se relaciona con mayor presencia de sintomatología postraumática; 3) la rumiación negativa tiene una mayor relación con la sintomatología postraumática que la reflexión y 4) la rumiación se relaciona en forma inversa con el estilo de afrontamiento represivo.

\section{MATERIAL Y MÉTODO}

Diseño transversal correlacional. La muestra fue obtenida por un muestreo no probabilístico y estuvo compuesta por 314 estudiantes universitarios (137 hombres y 177 mujeres), de la ciudad de Concepción, Chile, y cuyas edades fluctuaban entre los 19 a 33 años, con una media de $21.71(\mathrm{DE}=2.14)$. Se seleccionaron cursos de $2^{\circ}$ y $4^{\circ}$ año de las carreras de Enfermería, Kinesiología, Ingeniería Civil Industrial y Educación Física, buscando que la muestra total tuviera grupos equivalentes de hombres y mujeres. Además, como criterio de inclusión todos ellos estuvieron expuestos al terremoto del 27 de febrero del $2010 \mathrm{y}$ al momento de la medición habían trascurrido nueve meses desde el evento o desastre natural. Todos los participantes firmaron un Consentimiento informado.

Este trabajo contó con la aprobación del Comité de Ética de la Facultad de Enfermería de la Universidad San Sebastián.

\section{Variables e instrumentos}

Estrés postraumático: Para evaluar la sintomatología postraumática se utilizó la versión española de Bobes et al. de la Escala de Trauma de Davidson (DTS) (19). Fue diseñada para valorar la frecuencia y severidad de los síntomas de estrés postraumático en sujetos que han sufrido un evento estresante. Sus 17 ítemes se basan en los criterios DSMIV. Cada ítem se evalúa mediante una doble escala: una de frecuencia y otra de gravedad. La suma de ambas escalas conforma la puntuación total de sintomatología postraumática. El rango total de la escala es de 0 a 136. A mayor puntuación mayor severidad y/o frecuencia. La escala es autoadministrada y el marco de referencia temporal es la semana anterior.

En la validación española el alfa de Cronbach obtenido fue de 0.90 . La validación española también obtuvo valores adecuados de validez concurrente y discriminante (19). En el presente estudio se obtuvo un alfa de 0.95 para la escala total.

Ansiedad: Para evaluar ansiedad se empleó la Escala de Ansiedad Manifiesta de Taylor (TMAS). Esta escala es ampliamente utiliza- 
da como un indicador general de la ansiedad como un rasgo de la personalidad. Consta de 50 ítemes que se contestan como Verdadero o Falso (20).

Se ha descrito un alfa de Cronbach de 0.82 para TMAS. En test-retest la fiabilidad, medida en un intervalo de 7 meses, indica una estabilidad de 0.66 (10). En este estudio $(\mathrm{n}=314)$ la consistencia interna de la Escala de TMAS obtuvo un alfa de Cronbach de 0.90 . Se eliminaron los ítemes 3 "Soy casi tan nervioso como las demás personas", el ítem 9 "Me ruborizo tan a menudo como los demás", el ítem 15"Mis manos y mis pies están generalmente calientes", el ítem 42 "Soy más consciente de mí mismo que la mayoría de las personas" y el ítem 43 "Soy el tipo de persona que toma las cosas con esfuerzo", por correlacionar negativamente con el puntaje total.

Deseabilidad social: Para evaluar este constructo se empleó la Escala de deseabilidad social de Marlowe y Crowne (SDS) es reconocida como el instrumento más popular y utilizado para medir la deseabilidad social. Consta de 33 ítemes que contienen frases relacionadas con actitudes personales, a las cuales se contesta $\mathrm{V}$ o $\mathrm{F}$.

La consistencia interna de la escala en distintos estudios se mueve entre 0.75 y 0.85 $(10,21)$. Se han obtenido valores de estabilidad temporal tras un intervalo de un mes de 0.89 (21). Su aplicación en Chile con estudiantes universitarios ha reportado adecuados niveles de consistencia interna (alfa de Cronbach 0.71) y estabilidad temporal (22). Una adaptación realizada en Chile, el MCSD-10, muestra una consistencia interna de 0.60 (23).

Afrontamiento represivo: Para la valoración del afrontamiento se empleó el Índice de Autorregulación Emocional (ISE) propuesto por Mendolia (24). Esta se diferencia de la forma de valoración clásica del afrontamiento represivo en que permite obtener una medida continua de esta dimensión. Se obtiene a través de un algoritmo que permite combinar los puntajes de la escala de ansiedad y deseabilidad social previamente descritas. La medida clásica tipifica grupos de personas, distinguiendo una categoría de represores, donde se incluyen a las personas bajas en ansiedad y altas en deseabilidad social. Puntuaciones de ISE elevadas indican mayor tendencia al estilo afrontamiento represivo (24). Complementariamente, se empleó también el procedimiento tradicional de evaluación del afrontamiento represivo. Para simplificar la presentación estos análisis no son presentados pero sus resultados fueron equivalentes a los obtenidos con el empleo del ISE.

Rumiación: Se utiliza la versión abreviada de la Ruminative Response Scale Esta versión excluye todos los ítemes de la original escala RRS que podría solaparse con sintomatología depresiva. Se utiliza la traducción al español de Cova et al. (13). Análisis factoriales confirmatorias indican que esta escala está compuesta por dos factores, de 5 ítemes cada uno: Reflexión y Rumiación Negativa. Se agregó un ítem a la escala de reflexión ("Trato de descubrir por qué me siento así") y dos ítemes a la de Rumiación Negativa ("Le doy vueltas en mi mente una y otra vez a las cosas malas que me han pasado", "Recuerdo todas las veces en que me pasan cosas malas"). Estos ítemes han sido estudiados por Cova et al. y han mostrado un comportamiento apropiado (25).

El alfa obtenido en la Escala de Reflexión fue de 0.75 en la primera evaluación y de 0.80 en la de seguimiento, y en la Rumiación Negativa fue de 0.80 y 0.82 . En este estudio la Escala de Respuesta Rumiativa Total (RRS) fue de 0.88 , la Escala de Reflexión 0.76 y la Escala de Rumiación Negativa 0.85 .

Se empleó para los análisis bivariados correlación producto momento de Pearson y, para el multivariado, regresión múltiple de tipo jerárquico, donde la variable criterio fue estrés postraumático y las variables predictores, en el primer paso, sexo y edad, y, en el 
segundo, afrontamiento represivo, reflexión y rumiación negativa.

\section{RESULTADOS}

En la Tabla 1 se presentan los resultados descriptivos de las variables evaluadas. En la Tabla 2 se comparan los promedios en las prin- cipales variables de estudio entre hombres y mujeres.

Un 7\% de la muestra mostró sintomatología de TEPT sobre el punto de corte habitual de la escala de Davidson (40 puntos). Un $10.2 \%$ de las mujeres presentaron sintomatología sobre el punto de corte; en cambio, sólo un $2.9 \%$ de los hombres estaban en esta condición (diferencia significativa, $\mathrm{X}^{2}=6.23$; $\mathrm{p}<0.05)$.

Tabla 1. Estadísticos descriptivos de las variables del estudio $(\mathrm{n}=314)$.

\begin{tabular}{|c|c|c|c|c|}
\hline Variable & Mínimo & Máximo & Media & D. Estándar \\
\hline Edad & 19 & 33 & 21,71 & 2,14 \\
\hline Estrés Postraumático Total & 0 & 103 & 12,50 & 16,30 \\
\hline Estrés Gravedad ${ }^{\mathrm{a}}$ & 0 & 57 & 6,90 & 9,16 \\
\hline Estrés Frecuencia ${ }^{\mathrm{b}}$ & 0 & 50 & 5,60 & 7,92 \\
\hline Afrontamiento Represivo ISE & 0 & 58 & 27,88 & 11,25 \\
\hline Rumiación Total & 0 & 38 & 14,57 & 8,07 \\
\hline Rumiación Negativa $^{c}$ & 0 & 21 & 8,18 & 4,99 \\
\hline Rumiación Reflexiva $^{\mathrm{d}}$ & 0 & 18 & 6,39 & 3,94 \\
\hline
\end{tabular}

a: Estrés Gravedad es una subvariable de Estrés Postraumático Total

b: Estrés Frecuencia es una subvariable de Estrés Postraumático Total

c: Rumiación Negativa es una subvariable de Rumiación Total

d: Rumiación Reflexiva es una subvariable de Rumiación Total

Tabla 2. Diferencias de estrés postraumático total, afrontamiento represivo ISE y rumiación, según género.

\begin{tabular}{|c|c|c|c|c|c|}
\hline \multirow{2}{*}{ Variables } & \multicolumn{2}{|c|}{ Hombres $(n=137)$} & \multicolumn{2}{|c|}{ Mujeres $(n=177)$} & \multirow[b]{2}{*}{$t$} \\
\hline & Media & DS & Media & DS & \\
\hline Estrés Postraumático Total & 8.05 & 10.8 & 15.94 & 18.8 & $4.66^{\star * *}$ \\
\hline Estrés Gravedad & 4.31 & 5.8 & 8.9 & 10.6 & $4.85^{\star * *}$ \\
\hline Estrés Frecuencia & 7.03 & 9.0 & 3.74 & 5.6 & $3.95^{\star * *}$ \\
\hline Afrontamiento Represivo ISE & 30.91 & 10.06 & 25.54 & 11.57 & $-4.31^{\star * *}$ \\
\hline Rumiación Total & 12.83 & 7.67 & 15.92 & 8.14 & $3.41^{\star * *}$ \\
\hline Rumiación Negativa & 7.92 & 4.79 & 8.86 & 5.04 & $2.79^{* * *}$ \\
\hline Rumiación Reflexiva & 5.53 & 3.58 & 7.05 & 4.07 & $3.43^{* * *}$ \\
\hline
\end{tabular}

${ }^{\star} p<0.05,{ }^{* *} p<0.01,{ }^{* * *} p<0.001$

Como se puede apreciar, existen diferencias de género significativas para estrés postraumático y sus subescalas de frecuencia y gravedad y para rumiación y sus componentes: rumiación negativa y reflexiva. En relación al afrontamiento represivo ISE, también 
las diferencias resultan ser estadísticamente significativas.

En la Tabla 3 se presenta el coeficiente de correlación producto-momento de Pearson entre las variables consideradas en este estudio.

Como se desprende del análisis de la Tabla 3 , se observan correlaciones moderadas a altas entre las variables consideradas. Aquellos participantes que presentan mayores niveles de estrés postraumático total se caracterizan por exhibir menores niveles de afrontamiento represivo ISE, $r(314)=-0.45, p<0.001 \mathrm{y}$ mayores niveles de rumiación total, $r(314)=$ $0.40, p<0.001$. Los participantes que muestran mayores niveles de afrontamiento represivo ISE muestran menores niveles de rumiación total, $r(314)=-0.57, p<0.001$.

Tabla 3. Matriz de correlaciones producto-momento de Pearson entre las variables estrés postraumático, afrontamiento represivo ISE y rumiación $(\mathrm{n}=314)$.

\begin{tabular}{|c|c|c|c|c|c|c|c|}
\hline & 1 & 2 & 3 & 4 & 5 & 6 & 7 \\
\hline 1: Estrés Postraumático Total & -- & & & & & & \\
\hline 2: Estrés Gravedad & $0.96^{\star *}$ & -- & & & & & \\
\hline 3: Estrés Frecuencia & $0.94^{\star *}$ & $0.82^{\star \star}$ & -- & & & & \\
\hline 4: Afrontamiento Represivo ISE & $-0.45^{\star \star}$ & $-0.43^{\star \star}$ & $-0.42^{\star \star}$ & -- & & & \\
\hline 5: Rumiación Total & $0.40^{* *}$ & $0.39^{\star \star}$ & $0.36^{\star *}$ & $-0.57^{\star \star}$ & -- & & \\
\hline 6: Rumiación Negativa & $0.35^{\star \star}$ & $0.35^{\star \star}$ & $0.32^{\star *}$ & $-0.62^{\star \star}$ & $0.92^{\star \star}$ & -- & \\
\hline 7: Rumiación Reflexiva & $0.36^{\star *}$ & $0.37^{\star *}$ & $0.32^{\star *}$ & $-0.39^{\star *}$ & $0.87^{\star *}$ & $0.63^{\star *}$ & -- \\
\hline
\end{tabular}

${ }^{*}$ La correlación es significativa al nivel 0.01 (bilateral).

Con el fin de eliminar el posible efecto de factores de confusión, en particular el posible efecto de variables sociodemográficas y de evaluar la contribución independiente de cada una de las variables consideradas controlado el efecto de las otras, se realizó una regresión lineal múltiple jerárquica, en las que se analizó la posible relación del afrontamiento represivo y rumiación (reflexiva y negativa) (ingresadas como variables criterio), con la variable estrés postraumático (ingresada como predictor), controlando el efecto de las variables sociodemográficas. En esta regresión se ingresó en un primer paso las variables edad y sexo, mientras que en el segundo paso se introdujo el afrontamiento represivo y las dos formas de rumiación. Como los residuos no se distribuyeron normalmente, se debió normalizar la variable estrés postraumático total (con logaritmo raíz). La norma- lización no mostró cambios del análisis con la variable original, por lo que se presentan los análisis originales no normalizados.

La Tabla 4 incluye los dos pasos de la regresión realizada con la variable afrontamiento represivo ISE. Se aprecia en el primer paso que las variables sexo y edad son capaces de explicar un $5 \%$ de la varianza del estrés postraumático. El segundo modelo, donde se incluyeron afrontamiento represivo ISE, rumiación reflexiva y rumiación negativa, da cuenta de $26 \%$ de la varianza de la variable dependiente.

En la Tabla 5 se presenta el resultado del segundo paso del modelo de regresión para cada una de las variables. Se puede apreciar que el sexo $(\beta=-0.11)$, el afrontamiento represivo ISE $(\beta=-0.35)$ y la rumiación reflexiva $(\beta=0.21)$ son predictores significativos del estrés postraumático total. 
Tabla 4. Relación entre estrés postraumático total, rumiación negativa, rumiación reflexiva y afrontamiento represivo ISE.

\begin{tabular}{|c|c|c|c|c|c|c|c|}
\hline \multirow[t]{2}{*}{ Modelo } & \multirow[t]{2}{*}{$\mathbf{R}$} & \multirow[t]{2}{*}{$\mathbf{R}^{2}$} & \multirow{2}{*}{$\begin{array}{c}\mathbf{R}^{2} \\
\text { corregida }\end{array}$} & \multirow{2}{*}{$\begin{array}{c}\text { Error } \\
\text { típico de la } \\
\text { estimación }\end{array}$} & \multicolumn{3}{|c|}{ Estadísticos de Cambio } \\
\hline & & & & & $\begin{array}{c}\text { Cambio en } \\
\mathbf{R}^{2}\end{array}$ & Cambio en $\mathrm{F}$ & $\begin{array}{c}\text { Sig. del } \\
\text { cambio en F }\end{array}$ \\
\hline 1 & $0.24^{\mathrm{a}}$ & 0.05 & 0.05 & 15.87 & 0.05 & 9.650 & 0.000 \\
\hline 2 & $0.51^{\mathrm{b}}$ & 0.26 & 0.25 & 14.12 & 0.20 & 28.36 & 0.000 \\
\hline
\end{tabular}

Variable dependiente: Estrés postraumático total.

a. Variables predictoras: Sexo, Edad.

b. Variables predictoras: Sexo, Edad, Afrontamiento Represivo ISE, Rumiación Reflexiva, Rumiación Negativa.

Tabla 5. Coeficientes de regresión para las variables estrés postraumático total, afrontamiento represivo ISE, rumiación negativa y rumiación reflexiva.

\begin{tabular}{|lccccccc|}
\hline Variables predictivas & \multicolumn{2}{c}{$\begin{array}{c}\text { Coeficientes no } \\
\text { estandarizados }\end{array}$} & \multicolumn{2}{c}{$\begin{array}{c}\text { Coeficientes } \\
\text { estandarizados }\end{array}$} & & \multicolumn{2}{c|}{ Correlaciones } \\
\cline { 2 - 8 } & $\mathbf{B}$ & Error típ. & $\boldsymbol{B}$ & $\mathbf{t}$ & Sig. & Orden cero & Parcial \\
\hline Edad & 0.09 & 0.37 & 0.01 & 0.25 & 0.801 & -0.00 & 0.01 \\
Sexo & -3.83 & 1.67 & -0.11 & -2.28 & 0.023 & -0.24 & -0.12 \\
Afrontamiento & & & & & & & \\
Represivo ISE & -0.51 & 0.09 & -0.35 & -5.54 & 0.000 & -0.45 & -0.30 \\
Rumiación Negativa & -0.06 & 0.24 & -0.02 & -0.26 & 0.789 & 0.35 & -0.01 \\
Rumiación Reflexiva & 0.91 & 0.26 & 0.22 & 3.43 & 0.001 & 0.36 & 0.19 \\
\hline
\end{tabular}

\section{DISCUSIÓN Y CONCLUSIÓN}

Los resultados obtenidos respaldan tres de las cuatro hipótesis formuladas en la presente investigación.

En concordancia con la primera hipótesis del estudio, se observó una relación significativa e inversa entre afrontamiento represivo y estrés postraumático. Ello implica que las personas que informan mayores niveles de afrontamiento represivo presentan menores niveles de sintomatología postraumática.

Estos hallazgos son coincidentes con el estudio de Ginzburg et al. (9), donde se observaron tasas de prevalencia más bajas de trastorno de estrés agudo y de trastorno por estrés postraumático que observaron en una muestra de represores respecto a los otros grupos (defensivos y alta ansiedad), que habían sufrido un infarto agudo al miocardio. Por otra parte, Parker y McNally (10) también observan que este tipo de afrontamiento se asocia a mejor salud mental durante el duelo. Los autores piensan que esto podría estar mediado por la existencia de un sesgo interpretativo en los represores, relacionado con la deseabilidad social, que les haría dar un significado más positivo a sus pensamientos y emociones (10). En la misma dirección, Smeets et al. (11) observaron una relación negativa entre afrontamiento represivo en jóvenes universitarios con sintomatología postraumática. 
Respecto a la segunda hipótesis, los resultados obtenidos son consistentes con lo informado por investigaciones previas en el sentido que permiten concluir que aquellas personas que exhiben mayores niveles de rumiación tienden a informar mayores niveles de sintomatología postraumática $(12,16,26$, 27). Aunque con algunas excepciones (28) la mayoría de las pocas investigaciones en este tema muestran que la rumiación se asocia directamente con mayor presencia de sintomatología postraumática.

Sin, embargo, no se observó la relación planteada en la tercera hipótesis respecto a que se observaría una relación mayor entre rumiación negativa y sintomatología postraumática que con rumiación reflexiva. A nivel bivariado, la relación de ambos componentes de la rumiación con la sintomatología postraumática fue casi idéntica. En el análisis multivariado, sólo la rumiación reflexiva mostró una relación significativa. Sí se confirmó la última hipótesis referida a la existencia de una relación inversa entre afrontamiento represivo y rumiación.

La desaparición del efecto de la rumiación negativa podría deberse a la mayor varianza compartida de ésta con el afrontamiento represivo (la relación inversa entre afrontamiento represivo y rumiación negativa fue más acentuada que respecto a rumiación reflexiva). En general, los hallazgos disponibles apuntan a que sería la rumiación negativa la que tiene un rol más desfavorecedor en el desarrollo de problemas emocionales, aun cuando no son del todo consistente (14, 15). El estudio de Michael et al. (16) mostró relaciones del estrés postraumático sólo con rumiación negativa, en forma transversal y longitudinal (16). Se requiere más investigación a este respecto, y mayor clarificación de la distinción entre ambos tipos de rumiación.

Como era esperable dada la muestra considerada por el estudio, los niveles de sintomatología postraumática en la población evaluada eran moderados. Debe destacarse que si bien todos habían estado expuestos nueve meses atrás a un desastre natural, no todos fueron directamente $y$ significativamente afectados. Pese a ello, un 7\% de la muestra calificaba con un probable TEPT. Como es esperable, las variables estudiadas mostraron relación con diferencias de género. Los niveles de sintomatología postraumática observados en la muestra estudiada fueron, en concordancia con la mayoría de las investigaciones, más elevados en mujeres $(3,13,15,18)$. Lo mismo ocurrió respecto de la reflexión y rumiación negativa (14). Por el contrario, y también en el sentido esperado, los hombres mostraron promedios mayores de afrontamiento represivo.

Las implicaciones de este estudio pudieran dar cuenta de un nuevo enfoque respecto al afrontamiento represivo, no tan negativo como en los desarrollos originales de este concepto. Puede hipotetizarse que el afrontamiento represivo puede bloquear de ciertos procesos rumiativos desfavorables, que se constituyen en factores de riesgo para la aparición de TEPT.

Aunque los hallazgos recién descritos constituyen un aporte a la comprensión de las relaciones entre estrés postraumático, afrontamiento represivo y rumiación, éstos deben ser evaluados en el marco de las limitaciones del presente estudio. La naturaleza correlacional del diseño de investigación empleado no permite aseverar concluyentemente en cuanto a la dirección causal de las relaciones identificadas.

Otra limitación de importancia es la forma utilizada de evaluación del constructo de afrontamiento represivo. La forma de evaluación, basada en autoinforme exclusivamente, asume que la existencia de una baja ansiedad relacionada con una alta deseabilidad social implica un proceso represivo, pero este supuesto no es necesariamente válido. Se requieren líneas de investigación que utilicen parámetros fisiológicos que permitan comparar estos resultados con los obtenidos por los de autoinforme. En definitiva, se re- 
quiere una mejor delimitación del concepto de afrontamiento represivo, y de su relación con distintos procesos de autorregulación emocional, así como una diversificación de las forma de evaluarlo. Probablemente, en ciertas condiciones y circunstancias, y dominios particulares, un afrontamiento represivo puede tener efectos positivos; en cambio en otras, los resultados pueden ser distintos.

Diseños longitudinales permitirán asimismo dilucidar en forma más concluyente la naturaleza causal del afrontamiento represivo y la rumiación. Los resultados señalados son de interés para todos los profesionales implicados tanto en el tratamiento de los efectos de la exposición a situaciones traumáticas como de su prevención. Para los profesionales de la enfermería, frecuentemente implicados en el apoyo a personas y comunidades afectadas, los resultados obtenidos les permiten apoyar formas de afrontamiento constructivas y evitar asunciones apresuradas respecto de que toda forma de expresión de emociones negativas puede ser constructiva.

\section{REFERENCIAS}

1. Figueroa R, Marín H, González M. Apoyo psicológico en desastres: Propuesta de un modelo de atención basado en revisiones sistemáticas y metaanálisis. Rev Med Chil. 2010; 138: 143-151.

2. International Federation of Red Cross and Red Crescent Societies. Word Disas Rep 2000. Dordrecht, Holanda: Martinus Nijhoff.

3. Gaborit, M. Desastres y trauma psicológico. Pens Psicol. 2006; 2(7), 15-39.

4. Neria Y, Nandi A, Galea S. Post-traumatic stress disorder following disasters: a systematic review. Psychol Med. 2008. 1-14.

5. Cova F, Rincón P. El Terremoto y Tsunami del 27-F y sus Efectos en la Salud Mental. Ter Psicol. 2010; 28(2), 179-185.
6. American Psichiatric Association. DSM IV-TR: Manual diagnóstico y estadístico de los trastornos mentales. Texto revisado. Barcelona: Masson. 2002.

7. Weinberger D, Schwartz G, Davidson R. Low-anxious, high-anxious and repressive coping styles: Psychometric patterns and behavioral and physiological responses to stress. J Abnorm Psychol. 1979; 88, 369-380.

8. Myers L. The importance of the repressive coping style: findings from 30 years of research. Anxiety Stress Coping. 2010; 23(1), 3-17.

9. Ginzburg K, Solomon Z, Bleich A. Repressive Coping Style, Acute Stress Disorder, and Posttraumatic Stress DisorderAfter Myocardial Infarction. Psycho Med. 2002; 64, 748-757.

10. Parker H, McNally R. Repressive Coping, Emotional Adjustment, and Cognition in people Who Have Lost Loved Ones to Suicide. Suicide Life Threat Behav. 2008; 38 (6), 676-687.

11. Smeets T, Giesbrecht T, Raymaekers L, Shaw J, Merckelbach H. Autobiographical Integration of Trauma Memories and Repressive Coping Predict Post-Traumatic Stress Symptoms in Undergraduate Students. Clin Psychol Psychother. 2010; 17, 211-218.

12. Nolen-Hoeksema S, Jannay M. A Prospective Study of Depression and Posttraumatic Stress Symptoms After a Natural Disaster: The 1989 Loma Prieta Earthquake. J Pers Soc Psychol.1991; 61(1), 15-21.

13. Cova F, Rincón P, Melipillán R. Rumiación y presencia de sintomatología ansiosa y depresiva en adolescentes. Rev Mex de Psicol 2007; 24, 175-183.

14. Pimentel M, Cova F. Efectos de la Rumiación y la Preocupación en el Desarrollo de Sintomatología Depresiva y Ansiosa en Estudiantes Universitarios de la Ciudad de Concepción, Chile. Terap Psicol. 2011; 29(1): 43-52. 
15. Cova F, Rincón P, Melipillán R. Reflexión, Rumiación Negativa y Desarrollo de Sintomatología Depresiva en Adolescentes de Sexo Femenino. Terap Psicol 2009; 27(2), 155-160.

16. Michael T, Halligan SL, Clark DM, Ehlers A. Rumination in posttraumatic stress disorder. Depress Anxiety. 2007; 24 (5): 307-317.

17. Furnham A, Petrides KV, Spencer-Bowdage $S$. The effects of different types of social desirability on the identification of repressors. Pers Individ Dif. 2001; 33, 119-130.

18. Pérez C, Vicente B, Zlotnick C, Kohn R, Johnson J, Saldivia S, et al. Estudio Epidemiológico de sucesos traumáticos, trastorno de estrés postraumático y otros trastornos psiquiátricos en una muestra representativa de Chile. Salud Mental. 2009; 31, 145-153.

19. Bobes J, Calcedo-Barba A, García M, François M, Rico-Villademoros E, González MP, et al. y Grupo español de trabajo para el estudio del trastorno por estrés postraumático. Evaluación de las propiedades psicométricas de la versión española de cinco cuestionarios para la evaluación del trastorno de estrés postraumático. Actas Esp Psiquiatr. 2000; 28(4): 207-18.

20. Martínez Sánchez F. Alexitimia y estilo represivo de afrontamiento: ¿dos constructos emocionales diferentes o similares? Rev Elec de Psicol [Internet] 1998; 3(3) [citado 8 agosto 2010]. Disponible en: http://www.psiquiatria.com/psicologia/vol2num1/art_7.htm.
21. Ferrando P, Chico E. Adaptación y análisis psicométrico de la escala de deseabilidad social de Marlowe y Crowne. Psicoth. 2000; 12(3), 383-389.

22. Saiz J, Jerez A, Lucero C, Rojas P. Medición del prejuicio hacia el mapuche. Estud Social. 1988; 57(3), 111-127.

23. Saiz J, Díaz M, Ortega A, Oyarce P. Sexismo ambivalente en estudiantes universitarios chilenos: teoría, medición y diferencias de género. Rev de Psicol Social y Pers. 1998; 14(1), 1-14.

24. Mendolia M. An Index of self-regulation of emotion and the study of repression in social contexts that threaten or do not threaten self-concept. Emotion. 2002; 2(3), 215-232.

25. Cova F, Rincón P, Inostroza C, Melipillán R. Factores asociados prospectivamente a sintomatología depresiva en adolescentes tempranos de sexo femenino. Univ Psychol. 2012; 11, 3, 853-862.

26. Kirkegaard D, Martini M, Mehlsen MY, Zachariae R. The influence of rumination and defensiveness on negative affect in response to experimental stress. Scand J Psychol. 2004; 45, 253-258.

27. Ehring T, Frank S, Ehlers A. The Role of Rumination and Reduced Concreteness in the Maintenance of Posttraumatic Stress Disorder and Depression Following Trauma Cogn Cognit Ther Res. 2008; 32: 488-506.

28. Lommen M, Sanders A, Buck N, Arntz A. Psychosocial predictors of chronic PostTraumatic Stress Disorder in Sri Lankan tsunami survivors. Behav Res Ther. 2009; 47(1), 60-65. 